\title{
Vitiligo skin is imprinted with resident memory CD8 T cells expressing CXCR3
}

Katia Boniface ${ }^{1}$, Clément Jacquemin ${ }^{1}$, Christina Martins ${ }^{1}$, Anne-Sophie Darrigade ${ }^{1}$, Nesrine Boukhedouni ${ }^{1}$, Denis Thiolat ${ }^{1}$, Khaled Ezzedine ${ }^{2}$, Alain Taieb ${ }^{1}$, Julien Seneschal ${ }^{1}$

1Department of Dermatology and Pediatric Dermatology, National Center for Rare Skin diseases, INSERM U 1035, ATIPAVENIR, University of Bordeaux, France; 'Department of Dermatology, AP-HP, Hôpital Henri-Mondor, Créteil, France

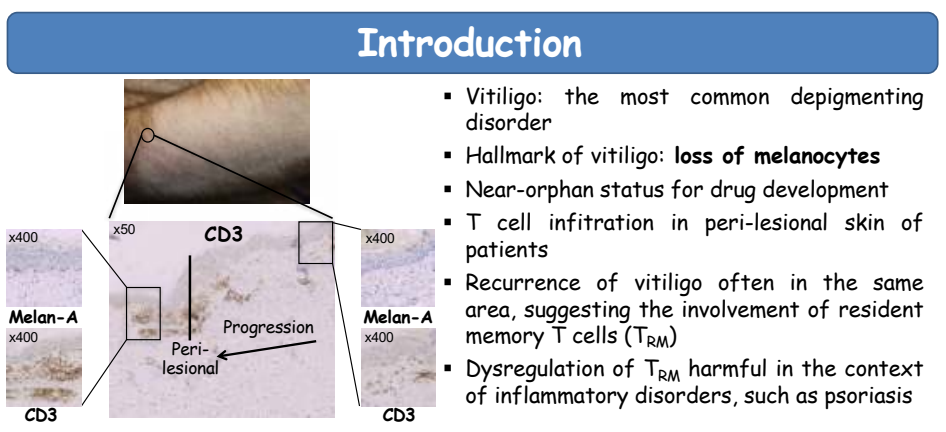

\section{Aim of the study}

To decipher the phenotype and function of skin infiltrating $T$ cells in vitiligo patients, and to compare such infiltrate to the one observed in psoriasis, the archetype of a skin inflammatory disorder

I. Vitiligo is enriched with populations of skin CD8 $T_{R M}$ cells expressing CXCR3

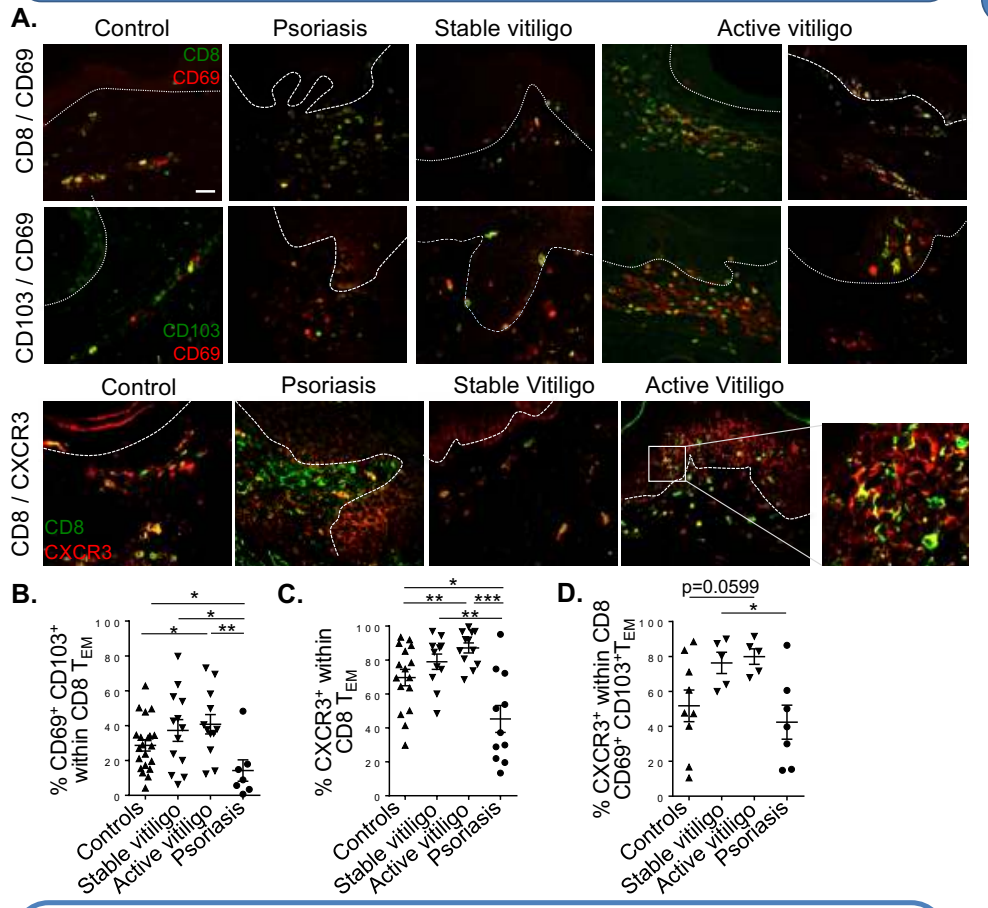

(A). Immunofluorescence microscopy analysis of CD8, CD69, CD103, and/or CXCR3 expression in skin biopsies from a healthy control, patients with stable or active vitiligo, or psoriasis. Scale bar represents $50 \mu \mathrm{m}$.

(B-D). T cells were isolated from controls healthy skin, perilesional skin of patients with stable or active vitiligo, and psoriasis skin. The proportion of. CD8 TEM cells expressing (B) CD69 and CD103 or (C) CXCR3 was determined by flow cytometry. (D) Frequency of CXCR3 cells within CD8 TRM cells. Each symbol represents one specimen. The mean \pm s.e.m. is shown. ${ }^{\star} p<0.05,{ }^{\star \star} p<0.01,{ }^{\star \star \star} p<0.001$,
II. Melanocyte-specific vitiligo skin CD8 $T_{E M}$ cells display increased expression of CXCR3

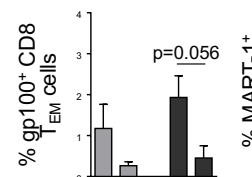

$R^{3} \beta^{3} R^{3} p^{3}$

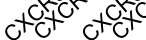

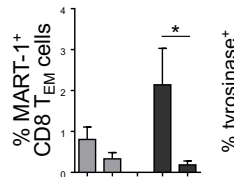

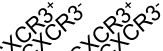

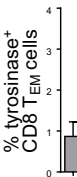

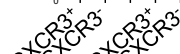

$\square$ Stable vitiligo

Active Vitiligo
The relative frequency of HLA-A2 restricted melanocyte-specific CD8 $T_{E M}$ cells within CXCR3 ${ }^{+}$ and $C X C R 3$ - subsets was evaluated by flow cytometry in the skin of patients with stable $(n=5)$ or active vitiligo $(n=5)$ using HLA-A2 pentamer loaded with the melanocyte peptides gp100, MART1 , or tyrosinase. The mean \pm s.e.m. is shown. ${ }^{*} p<0.05$.

III. Vitiligo skin CD8 $T_{E M}$ cells exhibit a skewed inflammatory cytokine profile with moderate cytotoxic activity

A.
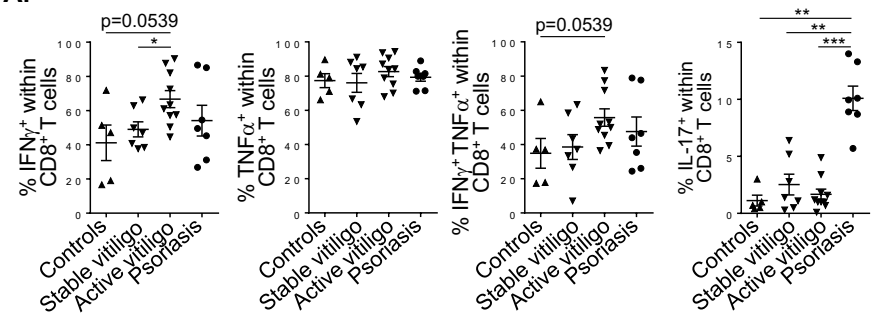

B.

c.
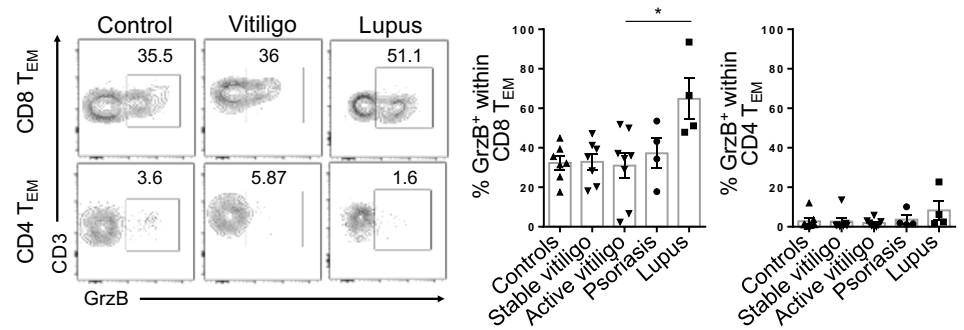

(A). T cells were isolated from controls healthy skin, perilesional skin of patients with stable or active vitiligo, and psoriasis skin. The proportion of IFN- $\gamma$, TNF- $\alpha$, IFN- $\gamma /$ TNF- $\alpha$ or IL-17 producing, skin-infiltrating CD8 T cells was evaluated by flow cytometry

(B-C). Expression of granzyme B was assessed on freshly isolated T cells from the skin of healthy controls, patients with a stable or active vitiligo, psoriasis, or lupus. Representative granzyme B staining is shown in panel (B). Each symbol represents one specimen. The mean s.e.m. is shown. ${ }^{\star} p<0.05,{ }^{\star \star} p<0.01,{ }^{\star \star \star} p<0.001$.

\section{Conclusion}

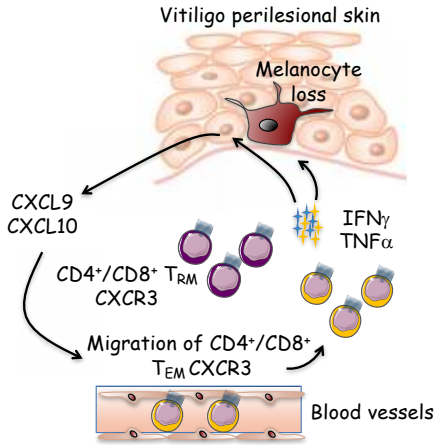

- Perilesional skin from vitiligo patients contains significant infiltrate characterized by a combination of CD103- and CD103 $C D 69^{+} C D 8 T_{R M}$ cells, reinforcing the concept of vitiligo as an immune memory skin disease

- Remaining CD8 $T_{R M}$ cells in patient perilesional skin could be an important mediator for disease flares

- Most skin infiltrating CD8 $T_{R M}$ cells in vitiligo bear expression of CXCR3

- Cytotoxicity is not the only mechanism involved in melanocyte disappearance in vitiligo and IFN- $\gamma$ and TNF- $\alpha$ produced by skin CXCR3+ $T$ cells could play a more important role

- Targeting $T_{R M}$ cells could be an attractive strategy in vitiligo

References : Bertolotti et al. Pigment Cell Melanoma Res. 2014; Boniface et al. Clin Rev Allergy Immunol 2017; Clark. Sci. Transl. Med. 2015; Le Poole et al. Am. J. Pathol. 1996; Picardo et al. Nat. Rev.Dis. Primers, 2015; Rashighi et al. Science Transl. Med. 2014: Van den Wingaard et al. Lab Invest 2000

\section{Université} BORDEAUX

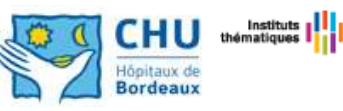

atip- avenir 